\title{
Geopolítica fronteriza y migraciones en la Rusia postsoviética ${ }^{1}$
}

\author{
SiLVIA MARCU \\ Investigadora « Ramón y Cajal » (RYC-2009-03834) \\ Instituto de Economía, Geografia y Demografia, CCHS, CSIC \\ silvia.marcu@cchs.csic.es
}

Unde venis et quo tendis?

Horace

Recibido: 24 de Mayo de 2010

Enviado a evaluar: 28 de Mayo de 2010

Aceptado: 24 de Febrero de 2011

\section{RESUMEN}

El artículo analiza la geopolítica de las fronteras de Rusia, después del desplome de la URSS. Se propone lograr un encuentro entre la geopolítica fronteriza y las migraciones, ámbitos de investigación separados en la actualidad (Gielis, 2009). La primera parte, presenta a Rusia como espacio de migración internacional, mientras que la segunda parte analiza el laberinto fronterizo de Rusia con la Unión Europea (UE), los países de la vecindad próxima, y los países de Asia Central y China. A continuación, se explora el significado de los movimientos migratorios transfronterizos, presentando la migración irregular y las rutas de migración de entrada, salida y de tránsito, que, procedentes de Asia y África atraviesan el territorio ruso para llegar a los países occidentales. Las conclusiones apuntan hacia una renovación de los estudios fronterizos para indagar en las sombras de la realidad migratoria del vasto y desconocido territorio de Rusia.

Palabras clave: Rusia, geopolítica fronteriza, migraciones internacionales, rutas de migración irregular, tráfico humano.

\section{Geopolitics of border and immigration in post-soviet Russia}

\begin{abstract}
This article examines the geopolitics of Russia's borders after the collapse of the USSR. It wants to make a meeting between the geopolitics of border and migration research fields are separated from each other (Gielis, 2009). The first part presents Russia as a space of global migration, while the second part analyzes
\end{abstract}

1 La realización de la investigación para este artículo ha sido posible gracias al Contrato "Ramón y Cajal" (RYC-2009-03834) concedido y cofinanciado por el Ministerio de Ciencia e Innovación de España y el Fondo Social Europeo. 
the labyrinth border between Russia and the European Union (EU), countries in the nearby vicinity, and the countries of Central and Eastern Asia and China. Then it explores the significance of cross-border migration, presenting irregular migration and migration routes of entry, exit and transit from Asia and Africa pass through Russian territory in search of the western dream. The findings point to a renewal of border studies, to look into the shadows of the migration situation of the vast and unknown territory of Russia.

Key Words: Russia, geopolitics of borders, international migrations, irregular migration routes, human trafficking.

\section{Géopolitique des frontières et des migrations de la Russie post soviétique}

\section{RESUMÉ}

Cet article examine la géopolitique des frontières de la Russie après l'effondrement de l'URSS. Il veut faire une réunion entre la frontière et les champs de recherche sur la migration sont séparés les uns des autres (Gielis, 2009). La première partie présente la Russie comme un espace de migrations au niveau international, tandis que la seconde partie analyse la frontière entre le labyrinthe de la Russie et l'Union Européenne (UE), les pays dans les environs immédiats, et les pays d'Asie centrale et orientale et Chine. Explore, ensuite l'importante de la migration transfrontalière, en présentant la migration irrégulière et les voies de migration de transit en provenance d'Asie et d'Afrique passent par le territoire russe à la recherche du rêve occidental Les résultats soulignent un renouvellement des études des frontières, de regarder dans l'ombre de la situation migratoire du territoire vaste et inconnu de la Russie.

Mots clé: Russie, les frontières géopolitiques, les migrations internationales, les voies de migration irrégulière, le trafic humain.

\section{INTRODUCCIÓN: DE LA GEOPOLÍTICA FRONTERIZA A LA GEOPOLÍTICA DE LAS MIGRACIONES}

Las fronteras representan uno de los más importantes temas de investigación en la geografía política (Paasi, 2003). Tradicionalmente, se han definido como elementos materiales del paisaje, y se han percibido como líneas que separan las "estructuras del poder" del Estado soberano, que ofrecen oportunidades tanto para la cooperación como para la discordia. Sin embargo, las fronteras representan más que unas "marcas" de los límites de la soberanía estatal (Prescott, 1987:80).

El territorio ruso es un caso de estudio idóneo, para destacar la diferencia entre el límite y la frontera, que, tal como señaló Van Houtum (2005:672) es más compleja, al ser entendida como un lugar a través del cual se comunican las diferencias socio-espaciales.

En Rusia, después de la desmembración de la URSS, en 1991, la amplia frontera euroasiática se vio fragmentada, transformándose en zonas fronterizas de diferentes características. Donde antes había un territorio complejo, salpicado de distintos espacios socio-culturales, en la actualidad se crearon fronteras, tanto penetrables como impenetrables, a través de las cuales, según las condiciones geopolíticas creadas, Rusia mantie- 
ne o no, relaciones con los países próximos, o más alejados. La existencia de las fronteras conduce a la creación de áreas geopolíticas en las que se producen intensos movimientos regulares e irregulares de personas de distintas identidades (Marcu, 2007).

En la literatura científica reciente (Hirsch, 2000, Franklin y Widdis, 2004), las identidades nacionales fueron percibidas, principalmente, como productos de construcción de la nación dirigidos por grupos de elite que buscan legitimar poder y estatus. Especialmente, en el contexto de la suavización de la antigua división entre el Este y el Oeste, la definición nacional de las fronteras vinculada a las identidades, no desapareció. Al contrario, surgió una fuerte tendencia de (re)-nacionalización de las fronteras, proceso que se relacionó con la formación de nuevas comunidades políticas (Eskelinen et al., 1999).

En la literatura sobre las migraciones, cuando se las considera, las fronteras suelen ser percibidas como límites estatales, líneas físicas y políticas que separan los territorios de los Estados. En esta condición, los límites se transforman en obstáculos y, por tanto, se crea una barrera de incompatibilidad entre las fronteras y las migraciones. Pero las fronteras tienen su gran influencia sobre los procesos migratorios y la movilidad (Van Houtum y Van Naerssen, 2002).

A pesar de la existencia de publicaciones sobre Rusia en materia de migración internacional, las que se refieren a la relación entre migraciones y fronteras, son casi inexistentes. A menudo, las investigaciones hacen hincapié en la inmigración de tránsito (Zaionchkovskaya, 1994), en las características socio-demográficas de la población activa extranjera (nacionalidad y profesión de los inmigrantes) (Balatsky, 1995), o en las características laborales de la mano de obra irregular extranjera (Zacharova et al., 1994).

Los estudios migratorios sobre las repúblicas de la antigua URSS (Vitkovskaya (1996), Tishkov (1996), Pilkington (1998), Heleniak (2002) y Flynn (2004) se centran en las presiones de la repatriación étnica de los rusos. Del mismo modo, una serie de trabajos académicos abordaron críticamente el concepto de "migración de retorno", señalando que "algunos migrantes mantienen fuertes y duraderos vínculos con sus países de origen, incluso si se incorporan a los países de reasentamiento" (Levitt et al., 2003: 565). En Rusia, específicamente, surgieron estudios que examinan los desafíos a los que se enfrentan los migrantes que regresan, al intentar integrarse en sus patrias "ancestrales". Tsuda (2003) sugiere que a pesar de la ausencia de diferencias con las poblaciones de la "tierra natal" (homeland), los migrantes que regresan, a menudo, se convierten en una nueva minoría debido a las características culturales distintas, adquiridas como resultado de la interacción con las sociedades de donde regresan.

Todos estos aspectos de la movilidad en las ex repúblicas soviéticas, a pesar de la falta de conocimiento y análisis, están vinculados a la frontera, como instrumento geopolítico fundamental en el proceso migratorio. En este marco, el artículo se propone realizar una incursión analítica por la geopolítica migratoria de las fronteras de la Rusia post-soviética. El trabajo analiza los cambios en la movilidad humana de acuerdo con los cambios geopolíticos producidos. Después de presentar Rusia como nuevo espacio global de migración y sus límites en el espacio post-soviético, se analizan las fronteras de Rusia con los países de la UE, con los vecinos próximos y con los países de Asia Central y del Sur. 
A continuación, se analizan las migraciones transfronterizas a través de la inmigración irregular y las rutas migratorias que llegan a Rusia y transitan su territorio en el camino hacia Occidente. Las conclusiones apuntan hacia una renovación de los estudios fronterizos y a la necesidad de vincularlas a las migraciones internacionales.

\section{RUSIA: UN NUEVO ESPACIO GLOBAL DE MIGRACIONES}

El colapso soviético significó el fin del sistema bipolar y el inicio de la nueva fase en las relaciones internacionales. Como consecuencia de los cambios ocurridos, Rusia apareció en el escenario de las migraciones internacionales a finales de los años 80 , y a lo largo de las últimas dos décadas se convirtió en un actor activo en los procesos migratorios globales, como país receptor, emisor y de tránsito (Bater, 1996). La crítica situación demográfica, la geopolítica de Rusia, su posición económica más estable en comparación con otros países de la Comunidad de Estados Independientes (CEI) y otros países del sudeste asiático, la debilidad de su legislación migratoria, las fronteras porosas con los países de la CEI y la presencia de un gran número de diásporas étnicas en su territorio, son indicadores de que seguirá siendo un país receptor de inmigrantes, durante un largo período de tiempo.

Además, el vasto espacio de la ex URSS mediante sus importantes recursos energéticos, se ha vuelto más disponible para diversas formas de cooperación, pero al mismo tiempo, se ha convertido en una importante fuente de inestabilidad a escala global. Debido a las relaciones económicas y a la interdependencia política y étnicocultural de los Estados post-soviéticos ${ }^{2}$, estos problemas tienen, a menudo, una dimensión transfronteriza que en muchos casos requiere tanto de coordinación interna, como de los agentes externos.

En estas condiciones, las nuevas fronteras adquieren diferentes significados. Por un lado, se persigue que garanticen un nivel suficiente de seguridad contra amenazas y desafíos como el narcotráfico, la migración irregular, la delincuencia transfronteriza y el extremismo. Por otra parte, se supone que la existencia de las fronteras no debe dañar los vínculos económicos, sociales, y culturales que se crearon principalmente en el periodo soviético.

En la mayoría de los casos, la estabilidad económica y social en las regiones fronterizas depende de la cooperación entre los Estados vecinos. Ivakhniouk (2006) resalta las siguientes características que resumen en gran parte el sistema de migración entre Rusia y los países de la Comunidad de Estados Independientes (CEI): lazos históricos y proximidad geográfica, transparencia de las fronteras (movimientos libres de visado), infraestructura común de transporte, facilidad para el cambio

2 El espacio post-soviético es una constelación de 14 Estados, agrupados en varias regiones: los tres países Bálticos (Estonia, Letonia y Lituania), Ucrania, Bielorusia y Moldavia, los países del Cáucaso (Armenia, Azerbaiyán y Georgia) y los cinco países de Asia Central (Kirguistán, Kazakhstan, Turkmenistán, Uzbekistán y Tajikistan) además de Rusia, que sigue siendo la gran potencia de la zona, tras el desplome de la URSS. 
psicológico (lenguaje, complementariedades del antiguo territorio común), interés común hacia el mercado de trabajo, migración irregular a gran escala y cooperación regional para la gestión migratoria coordinada.

Además de las migraciones que se producen, características para todo el sistema mundial, en la Rusia postsoviética nos encontramos tal como señalamos, con un tipo de migración particular: la inmigración de repatriación, o de retorno. Después del desplome soviético, en la década de 1990, alrededor de 25,3 millones de rusos se encontraron viviendo en los demás países de la CEI y en las tres repúblicas bálticas (Tishkov et al. 2005). Entre 1989 y 2008, con el telón de fondo de una importante crisis demográfica, Rusia recibió 12,9 millones migrantes procedentes de las repúblicas exsoviéticas (la actual Comunidad CEI y los Estados bálticos). Una gran parte de estos rusos tuvieron que trasladarse bajo algún grado de presión, muchos de ellos, incluso huyendo de los conflictos congelados del Cáucaso (Osetia del Sur, Abjasia). En Rusia, por lo tanto, se les llama "refugiados" o "migrantes forzosos" (también "reasentados forzosos"). Durante el mismo período, alrededor de 4,1 millones de personas emigraron desde Rusia hacia las ex repúblicas soviéticas que se habían independizado (OIM, 2008).

En la figura 1, se puede apreciar la dinámica migratoria de Rusia, las fluctuaciones existentes y la diferencia entre las salidas y las entradas en su territorio.

Figura 1. Dinámica de la inmigración en Rusia (entradas y salidas) 1996-2008.

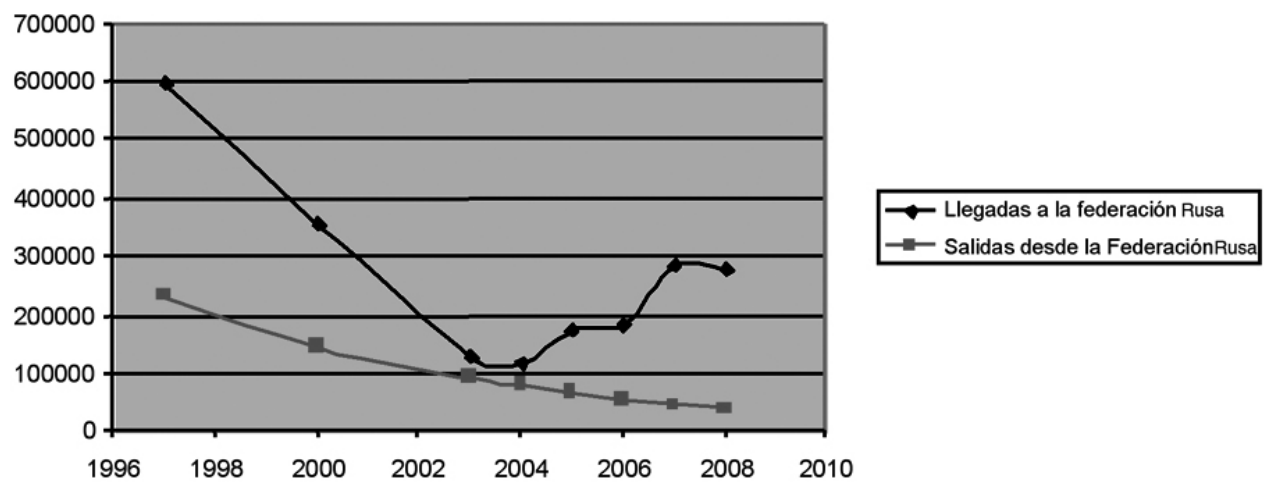

Fuente: Servicio Federal de las Migraciones de la Federación Rusa, 2009.

La frontera, instrumento que vincula la geopolítica y las migraciones internacionales, representa el símbolo y el hilo conductor para explicar los cambios en la movilidad humana en Rusia, en las últimas dos décadas. 


\section{UNA METAMÓRFOSIS GEOPOLÍTICA: LAS FRONTERAS RUSAS EN EL CONTEXTO DEL ESPACIO POST-SOVIÉTICO}

Geopolíticamente, las fronteras son consideradas como un atributo esencial del Estado nación. En el período soviético, la frontera del Estado era una férrea barrera que separaba el amplio territorio del resto del mundo. Adquirió importancia extrema como límite del mundo del homo sovieticus. La mayoría de los ciudadanos soviéticos, nunca cruzaron esa frontera que constituía el marco general de sus difíciles existencias (Heleniak, 2003). En las últimas décadas, después de su independencia, los países de la CEI han hecho progresos considerables hacia la consecución de la nación. Actualmente, la búsqueda de una identidad individual profundiza las diferencias entre los países. El trazado de fronteras nacionales, por lo tanto, está indisolublemente ligado a la conformación de la identidad de los Estados post-soviéticos. En el proceso de delineación de las nuevas fronteras, su función dominante es, en algunos casos, la de servir como barreras. Existen multitud de obstáculos a la libertad de movimiento y al contacto transfronterizo. Además, los nuevos Estados, al tener un desarrollo débil, ven limitada la financiación de la política social. En las repúblicas post-soviéticas, la mayor parte de la población vive por debajo del umbral de pobreza. El crecimiento de las disparidades socioeconómicas entre los Estados y el aumento de los problemas políticos, étnicos y sociales fortalecieron la función de barrera que cumplen las actuales fronteras (Voronina, 2006).

Por otra parte, las zonas fronterizas (borderlands) tienen que lidiar con la inmigración irregular, en mayor medida que en el interior los territorios (tratándose, ya sea de los trabajadores de temporada chinos en el Lejano Oriente, los refugiados de la región del Cáucaso, o los migrantes económicos de las regiones de Stavropol, Rostov y Krasnodar). Se estimó que en Rusia hay más de dos millones inmigrantes irregulares que penetran en el país, a través de las regiones fronterizas. También se señaló que existe una tendencia a formar zonas étnicamente pobladas (kazajos, armenios) dentro de las regiones fronterizas de Rusia, cuyos líderes ya plantearon la concesión de un estatuto especial de autonomía (Bagrandzhia, 2000). Generalmente, las nuevas fronteras de Rusia ${ }^{3}$ son zonas económicamente subdesarrolladas, situadas entre los límites nacionales y regionales de los nuevos Estados (OIM, op.cit). La situación se agrava por el hecho de que Rusia es un país relativamente pobre (su PIB per cápita es entre 7 y 10 veces menor que el de los países más desarrollados del mundo ${ }^{4}$. Además, la mayoría de las zonas fronterizas de los Estados vecinos, son las menos desarrolladas de sus territorios. Por otra parte, el deterioro de las relaciones de Rusia con algunos países de la CEI en la década de los noventa, que tuvo como una de las consecuencias

3 Las fronteras exteriores de Rusia son las más amplias del mundo (60.933 kilómetros), y el número de países limítrofes - 16 - es también el más extenso. Un 32,6\% de la población rusa vive en las zonas fronterizas. Por ello, las perspectivas de desarrollo de las regiones fronterizas representan una mezcla de oportunidades y desafíos.

4 Informe del Banco Mundial (2008). 
la introducción de los regímenes de visado, tuvo un fuerte impacto en la situación socio-económica en las regiones fronterizas. Las administraciones locales y regionales de las nuevas regiones fronterizas de Rusia lucharon por tener una influencia directa en el lugar de contacto o en la protección ofrecida por las fronteras. Se realizaron intentos para limitar los daños económicos y sociales causados por las nuevas fronteras, se intentó asegurar que la frontera es un factor positivo que impulsa el desarrollo económico regional; asimismo, se procuró aumentar los poderes de las autoridades locales y su participación en la cooperación transfronteriza.

Por lo tanto, las zonas fronterizas también ofrecen oportunidades: una de ellas consiste en que estas regiones, por lo general, reciben un trato preferencial por parte de los países vecinos. En tal sentido, China desea mantener su influencia en Primorsky krai, mientras que Finlandia tiene un interés particular en dar prioridad al desarrollo, a la vecina Karelia. En segundo lugar, las regiones fronterizas de Rusia son privilegiadas, ya que, además de los acuerdos bilaterales, tienen un estatuto legislativo especial, a nivel federal. Su estatuto favorece el desarrollo de los contactos con el extranjero, como los acuerdos transfronterizos de cooperación firmados entre el gobierno de Rusia y varios de sus vecinos como Finlandia (enero de 1992), Polonia (mayo de 1992), Kazajstán (enero de 1995), Ucrania (enero de 1995), Mongolia (enero de 1993) y China (mayo de 1994) ${ }^{5}$. Hasta finales de la década de los noventa, las autoridades locales y regionales de las nuevas regiones fronterizas trataron de persuadir al gobierno de la Federación Rusa, para aprobar una Ley federal especial para la cooperación en las regiones fronterizas. Tras las presiones de las regiones fronterizas, el gobierno de la Federación Rusa aprobó, en febrero de 2001, un nuevo concepto para el desarrollo de la cooperación transfronteriza, basado en la cooperación más estrecha (Vardomski, 2002).

\section{EL LABERINTO FRONTERIZO DE RUSIA}

Como vimos, las profundas transformaciones geopolíticas producidas en las dos últimas dos décadas, en el espacio post-soviético, crearon distintos mundos fronterizos. En este contexto, la cooperación transfronteriza (bilateral o multilateral) es todavía un fenómeno muy frágil en Rusia. Su vulnerabilidad se debe a varios factores: en primer lugar, las regiones fronterizas se encuentran, normalmente, en la periferia de las áreas "civilizadas". Por lo tanto, los países bálticos se consideran, culturalmente, "diferentes" a Rusia (Trenin, 1998). En el caso de la mayor parte de las fronteras rusas, (por ejemplo, Rusia-Bielorrusia; Rusia-Ucrania; Rusia-Kazajstán) se observa la debilidad de la función de frontera como barrera, y por tanto, en estas regiones, la inmigración está en aumento. En esos casos, las fronteras, al ser relativamente fluidas y flexibles, tienden a cosmopolitizarse (Rumford, 2006). Pero cuando su función de barrera es importante, (como ocurre en las fronteras situadas entre Rusia y

5 Existe, asimismo, un Acuerdo Intergubernamental (entre Rusia, Bielorrusia, Kazajstán y Kirguistán) de cooperación transfronteriza, firmado en febrero de 1999. 
Georgia o entre Rusia y China) la frontera obstaculiza la inmigración y se convierte en una infranqueable barrera.

En el caso que aquí analizamos, podríamos distinguir tres tipos de fronteras que separan a Rusia de las regiones y países vecinos (Figura 2).

Figura 2. Las Fronteras de Rusia.

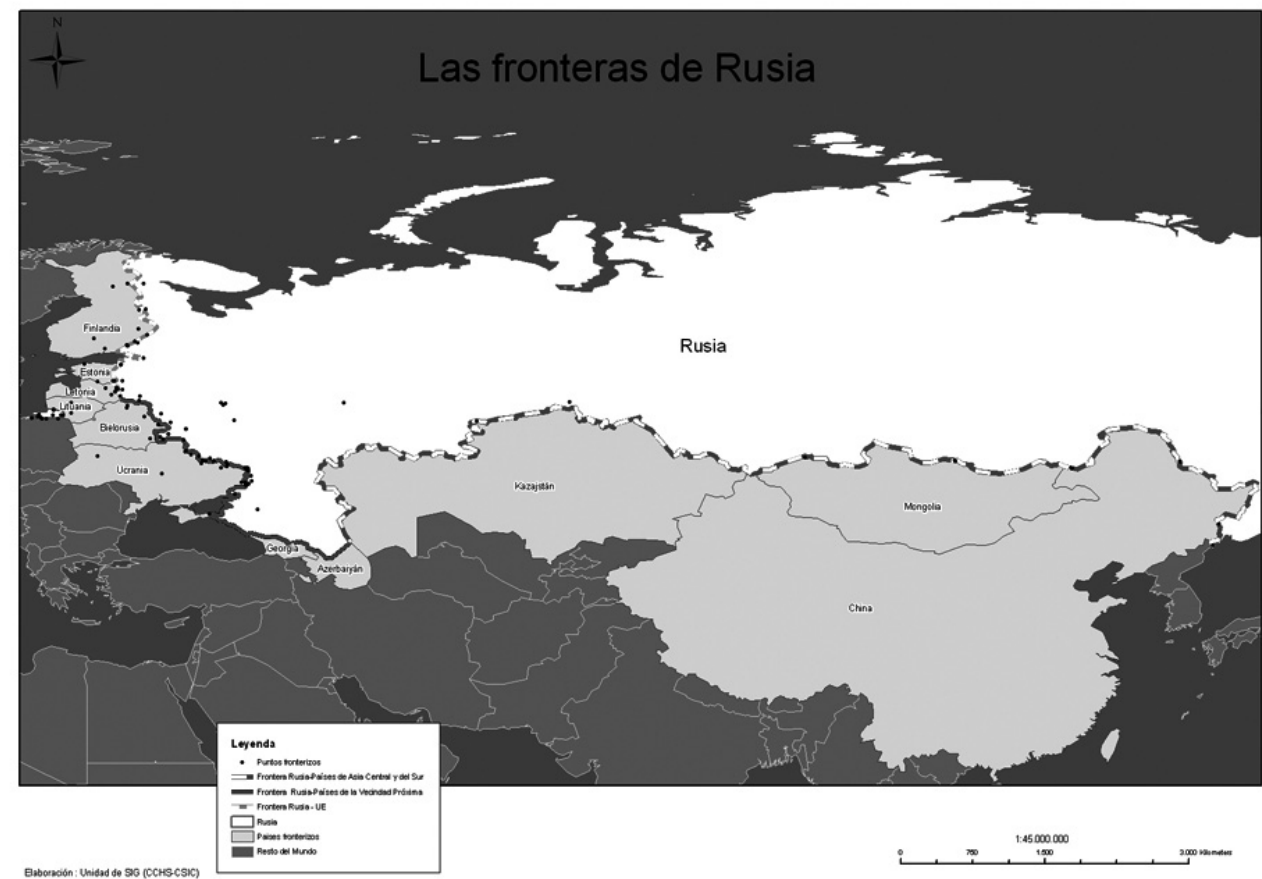

Fuente: Elaboración Laboratorio SIG CCHS, CSIC

1) La "frontera democrática" con la UE (Finlandia, Polonia y los Países Bálticos), que sitúa a Rusia a las puertas de la UE, o, incluso, en el interior, si nos referimos al enclave de Kaliningrado.

2) La frontera de "la vecindad próxima" que incluye el límite con Bielorrusia, Ucrania, Georgia y Azerbaiyán.

3) La amplia y porosa frontera de Europa Central y Asia del Sur, fronteriza con Kazajstán, China y Mongolia.

\subsection{LA "FRONTERA DEMOCRÁTICA"}

Después de la caída del telón de acero y la desintegración de la URSS, el mapa mental de Europa cambió drásticamente (O'Loughlin y van der Wusten, 1993). Estos cambios se hicieron más notables en el límite de las fronteras entre Rusia y la UE, especialmente en los países bálticos. 
Con Finlandia, que se unió a la UE en 1995, la frontera terrestre común entre Rusia y la UE llegó a tener 1.300 kilómetros de extensión. En 2000 se creó la euro región de Karelia, situada al noreste y dividida entre Finlandia (las regiones de Karelia del Norte y Karelia del Sur) y Rusia (la república de Karelia y el Oblast de San Petersburgo) (Paasi, 1999). Según la Comisión Europea se identificaron cuatro prioridades para la cooperación transfronteriza entre Finlandia y Rusia: apoyar el desarrollo de las redes de infraestructuras, especialmente en los pasos fronterizos, promover la promoción de la protección del medio ambiente; apoyar el desarrollo económico y el fomento de los pequeños proyectos establecidos por las autoridades regionales y locales (Comisión Europea, 2009) ${ }^{6}$.

Tras el colapso de la Unión Soviética, la frontera administrativa entre Rusia y Estonia, Lituania y Letonia se convirtió en una frontera del Estado nacional. De acuerdo con Giddens, (1987) el proceso de formación de los Estados modernos está estrechamente relacionado con la solidificación de sus fronteras nacionales. Las ex fronteras que se consideraban como una zona de transición, se convirtieron en el caso de los países bálticos en bordes sólidos, en zonas de separación de los Estados nacionales.

La gran ampliación de la UE de 2004, que incluyó a los tres países bálticos, aumentó la longitud de la frontera común a casi 2.000 kilómetros, y en relación con estos países, se centró en dos aspectos: por un lado, se propuso convertir Kaliningrado en un enclave ruso de la UE, y por el otro, quiso cambiar el régimen de fronteras entre Rusia y los países bálticos. De esta manera, la ampliación no sólo cambió el contenido del discurso en torno a las fronteras de la UE con Rusia, sino que significó también una ampliación de la agenda política. Por un lado, las normas y prácticas de Schengen se introdujeron en la frontera con Lituania, creando dificultades prácticas para el tránsito desde Rusia hacia Kaliningrado. Por otro lado, las personas que viven en la frontera Ruso-Estonia, (incluyendo una pequeña minoría de los indígenas de $\mathrm{Seto}^{7}$ ), perdieron el privilegio de viajar a Estonia en virtud de un régimen especial simplificado. Después de tensas negociaciones con Rusia, (en particular por la cuestión de Kaliningrado) entre 2000 y 2002, la UE tomó la decisión de ajustar el régimen de fronteras para atender a las necesidades locales. En la actualidad, los ciudadanos rusos pueden viajar a Kaliningrado a través del territorio de Lituania, con un documento de tránsito ferroviario (un solo viaje en tren) y documento de tránsito (varios viajes en coche). Además, Estonia accedió a proporcionar a los ciudadanos de Rusia que viven en las zonas adyacentes, visados de entrada múltiple.

6 La Política de Vecindad Europea en acción: puesta en marcha de programas de cooperación transfronterizacon Rusia.IP/09/1727 http://europa.eu/rapid/pressReleasesAction.do?reference=IP/09/1727\&format $=$ HTML\&aged $=0$ \&language $=\mathrm{EN} \&$ guiLanguage $=\mathrm{en}$

7 Setos (setoq) son una minoría étnica y lingüística autóctona del sureste de Estonia y noroeste de Rusia. Son en su mayoría cristianos ortodoxos Seto. Su dialecto, que algunos consideran una lengua independiente - la lengua de Seto - pertenece al grupo báltico-finés de las lenguas fino - húngaras. Seto está en busca de un mayor reconocimiento, siendo tradicionalmente considerado un dialecto de la lengua estonia. 
Las visas se conceden cada año y la emisión está regulada por la necesidad del solicitante de viajar a Estonia con frecuencia por motivos religiosos o familiares.

Por otra parte, la UE desarrolló su relación fronteriza con Rusia, de acuerdo con la vieja fórmula de promoción de la cooperación transfronteriza. Entre 2003 y 2004, otras dos Eurorregiones se establecieron en Kaliningrado: una entre Rusia - Polonia y Lituania - Letonia y otra entre Rusia y Estonia.

Asimismo, en el caso de las fronteras con los países bálticos, somos testigos de la existencia de zonas fronterizas donde se crearon identidades mixtas. Este es el caso de la frontera entre Letonia, Estonia y Rusia, en la región de Pechory, distrito Piskov, donde la frontera representa un factor importante en la vida cotidiana de la población situada a ambos lados de la misma (Kononenko, 2004). El problema de la identidad de los Estados bálticos, implica, pues, la existencia de una frontera social y psicológica para los ciudadanos de las zonas fronterizas.

En resumen, los principales problemas de la frontera externa entre Rusia y la UE se hacen más visibles en los pasos fronterizos, en relación con su infraestructura, acceso a la información, la comunicación entre los viajeros y el personal empleado, la cooperación entre las autoridades fronterizas, locales, municipales y estatales.

Tal como se puede apreciar en la tabla 1, en los últimos años, las llegadas de inmigrantes procedentes de países de la UE a Rusia, disminuyeron constantemente.

Tabla 1. Llegadas a la Federación Rusa, a través de la frontera con la UE (datos oficiales)

\begin{tabular}{|c|c|c|c|c|c|c|c|c|}
\hline Países UE & 1997 & 2000 & 2003 & 2004 & 2005 & 2006 & 2007 & 2008 \\
\hline Estonia & 3483 & 786 & 445 & 446 & 432 & 347 & 508 & 476 \\
\hline Letonia & 5658 & 1785 & 906 & 819 & 726 & 766 & 887 & 716 \\
\hline Lituania & 1785 & 945 & 535 & 339 & 360 & 371 & 537 & 455 \\
\hline Finlandia & 140 & 83 & 125 & 141 & 129 & 137 & 172 & 174 \\
\hline Suecia & 32 & 14 & 22 & 16 & 23 & 32 & 39 & 30 \\
\hline Polonia & 247 & 61 & 39 & 48 & 55 & 48 & 96 & 100 \\
\hline
\end{tabular}

Fuente: Servicio de Migraciones de la Federación Rusa, (Федеральная Миграционная Служба), 2009

\subsection{PUENTES Y ABISMOS: LA FRONTERA CON LOS PAÍSES DE LA "VECIN- DAD PRÓXIMA"}

La frontera entre Rusia y los países de la vecindad próxima se creó después del colapso de la URSS y la independencia de los países que formaron parte del antiguo espacio soviético (1991).

En un primer término, se sitúa la frontera "abismal" que comparte Rusia con Georgia, cargada de tensiones políticas, y vinculada a las férreas políticas de seguridad de ambos países. Las raíces del abismo están en los conflictos ideológicos y étnicos que surgieron en las últimas dos décadas.

Como resultado, en 2000, el gobierno ruso introdujo el régimen de visados para los nacionales de Georgia, mientras que los demás países de la CEI continuaron disfrutando de un movimiento libre hacia Rusia.

El paso fronterizo, en el control de Alta Lars en las montañas del Cáucaso, estuvo cerrado desde 2006, cuando aumentaron las tensiones entre los dos países, en 
parte, debido a los esfuerzos del presidente georgiano Saakasvili, por estrechar sus relaciones con los Estados Unidos.

En 2008, la disputa territorial influyó en la movilidad de las personas, sobre todo, con el estallido de la guerra después de que Georgia tratara de recuperar Osetia del Sur, un enclave separatista que tiene estrechos vínculos con Moscú, y cuando las tropas rusas respondieron invadiendo Georgia. Tras año y medio de cierre total y de ausencia de relaciones diplomáticas, Rusia y Georgia reestablecieron la apertura de uno de sus pasos fronterizos, en marzo de $2010^{8}$.

Por el contrario, la frontera con otro país vecino, Bielorrusia, es simbólica, prácticamente inexistente. Los ciudadanos de Belarús no tienen necesidad de visado para entrar en Rusia. En octubre de 2007, Rusia y Belarús, junto a Kazajstán firmaron un paquete de acuerdos sobre la base jurídica de la unión aduanera, incluyendo la formación de una comisión y una unión aduanera común. Mientras tanto, a principios de 2010, El Ministerio de Asuntos Exteriores de Letonia firmó un acuerdo de fronteras con Bielorrusia y Rusia para definir un punto fronterizo común entre los tres países.

Por su parte, la frontera entre Rusia y Ucrania constituye el límite europeo más amplio entre los países de la antigua URSS. Los pasos fronterizos entre Rusia y Ucrania conectan con multitud de países asiáticos cuyos ciudadanos se dirigen a los países de la UE. Ello contribuye a que esta zona de frontera entre Ucrania y Rusia sea el regulador clave de los flujos transfronterizos de Eurasia.

Dentro de las regiones fronterizas existentes entre Rusia y Ucrania se concentra parte significativa del potencial industrial: más del $40 \%$ de la producción industrial de Ucrania y un $6 \%$ de la producción industrial rusa se elabora allí.

Entre todas las zonas fronterizas post-soviéticas, la frontera situada entre Rusia y Ucrania (al igual que en el caso ruso-bielorruso) es la zona en que se manifiesta en mayor grado el contacto y la relación etnocultural entre los ciudadanos rusos y ucranianos. Debido a la afinidad idiomática, la proximidad cultural, religiosa y la multitud de tradiciones y costumbres compartidas, los rusos y ucranianos que viven en las zonas fronterizas no se perciben los unos a los otros como extranjeros. A la comunicación social muy intensa, contribuyen, asimismo, los matrimonios interétnicos, o la migración de doble identidad (Golunov, 2007a). Por lo tanto, la cuestión fronteriza entre Rusia y Ucrania no representa sólo una disputa territorial. Inevitablemente, se convirtió en una relación compleja que atañe las cuestiones de Estado y de construcción de la nación, la creación de nuevas identidades nacionales post-soviéticas y el desarrollo de las naciones cívicas. Todos estos factores intervienen, pues, en el renacimiento de los mitos históricos y en la vuelta a la historia para "probar" el primer asentamiento en las tierras rusoucranianas (Kuzio, 1997).

8 En Marzo de 2010, se abrió el punto fronterizo Werhni-Lars (Верхний Ларс) para el transporte. 
Según los datos oficiales, la evolución de los inmigrantes de los países de la vecindad próxima se presenta en la tabla 2 :

Tabla 2. Llegadas de los países de la Vecindad Próxima a la Federación Rusa. (Datos oficiales)

\begin{tabular}{|c|c|c|c|c|c|c|c|c|}
\hline Países Vecindad Próxima & 1997 & 2000 & 2003 & 2004 & 2005 & 2006 & 2007 & 2008 \\
\hline Azerbaijan & 29878 & 14906 & 4277 & 2584 & 4600 & 8900 & 20968 & 23331 \\
\hline Armenia & 19123 & 15951 & 5124 & 3057 & 7581 & 12949 & 30751 & 35216 \\
\hline Bielorrusia & 17575 & 10274 & 5309 & 5650 & 6797 & 5619 & 6030 & 5865 \\
\hline Georgia & 24517 & 20213 & 5540 & 4886 & 5497 & 6806 & 10595 & 8806 \\
\hline Ucrania & 138231 & 74748 & 23418 & 17699 & 30760 & 32721 & 51492 & 49064 \\
\hline Moldavia & 13750 & 11652 & 6391 & 4816 & 6569 & 8649 & 14090 & 15519 \\
\hline
\end{tabular}

Fuente: Servicio de Migraciones de la Federación Rusa, Федеральная Миграционная Служба 2009

\section{3. "LAS FRONTERAS SON EL FILO DE LA NAVAJA"9: FRONTERAS CON LOS PAÍSES DE ASIA CENTRAL Y CHINA}

Por su apertura, elevada porosidad y las relativas buenas relaciones entre los dos países, la frontera entre Rusia y Kazajstán es muy importante para la movilidad humana que llega desde Asia Central y desde el hemisferio del sur. En todos los países de Asia Central, la sociedad ha sido políticamente inactiva o silenciada por el poder estatal, durante muchos años. Actualmente, la inseguridad está aumentando, en parte, debido a la proximidad a las zonas conflictivas de Afganistán y Pakistán. La infraestructura se está colapsando mientras que las economías más débiles están cayendo aún más. El relativo desarrollo de las economías de Rusia y Kazajstán, proporcionó a millones de habitantes de Asia Central el trabajo que no pudieron encontrar en sus países. Los datos oficiales de los migrantes llegados a Rusia de los países de la región de Asia Central (tabla 3 ) reflejan la situación de los inmigrantes regulares que entraron en Rusia. Pero entre 2004-2008, años de auge en el proceso migratorio, 800.000 ciudadanos de Kirguistán, 1,5 millones tayikos y 2,5 millones uzbecos emigraron a Rusia y a Kazajstán ${ }^{10}$, para trabajar en la construcción, agricultura

9 Referencia al reconocido texto de 1907 "Romans Lecture" sobre las fronteras, escrito por Lord Curzon of Celestin, Vizconde de India.

10 Las cifras son imprecisas debido a la baja calidad de la recogida de datos en los países de origen y destino. Lo que sigue representa los datos oficiales de los países emisores: Kirguistán - desde 300.000 (oficialmente) a 500,000-800,000 (estimaciones); Uzbekistán - de 250.000-300.000 (oficialmente) a 2-3 millones (estimación media), 5 - 6 millones (alta estimación); Tayikistán - 600,0000 (oficialmente) a 1,5 millones (alta estimación). Ver Informe del Grupo de Crisis, Tayikistán: de camino al fracaso, op. cit.; Kursad Aslan, "La migración laboral y sus posibles consecuencias para el Asia central", Asia Central-Cáucaso analistas, el 16 de abril de 2008, en www.cacianalyst.org/?q=node/4836; Донис Иван, "Айгуль Рыскулова: В России на заработках официально находятся около 300 тысяч трудовых мигрантов из Кыргызстана [Iván Donis," Aigul Ryskulova: 300.000 trabajadores inmigrantes de Kirguistán trabajan en Rusia oficialmente"], News Service, 6 de octubre de 2009, en http://24.kg/community/63748-ajgul-ryskulova-v-rossiina-zarabotkax-oficialno.html; Umida Hashimova, "La crisis financiera golpea Uzbekistán", Asia Central Cáucaso analistas, el 28 de enero de 2009, en www.cacianalyst.org/?q=node/5025; "Узбекистан: Кому выгодны новые меры властей по контролю за трудовой миграцией?” [“Uzbekistán: ¿Quién se beneficia de las normas del nuevo gobierno en el control de la migración laboral?’], Ferghana.ru, 2 de julio de 2007,a www.ferghana.ru 
y en los servicios de vivienda, por lo general, recibiendo sueldos muy bajos en sectores que la población local había rechazado.

Tabla 3. Llegadas a Rusia desde los países de Asia Central. (Datos oficiales)

\begin{tabular}{|c|c|c|c|c|c|c|c|c|}
\hline Asia Central & 1997 & 2000 & 2003 & 2004 & 2005 & 2006 & 2007 & 2008 \\
\hline Kazakhstan & 24517 & 20213 & 5540 & 4886 & 5497 & 6806 & 10595 & 8806 \\
\hline Kyrgyzstan & 13752 & 15536 & 6948 & 9511 & 15592 & 15669 & 24731 & 24014 \\
\hline Tajikistan & 23053 & 11043 & 5346 & 3339 & 4717 & 6523 & 17309 & 20717 \\
\hline Turkmenistán & 16501 & 6738 & 6299 & 3734 & 4104 & 4089 & 4846 & 3962 \\
\hline Uzbekistán & 16501 & 6738 & 6299 & 3734 & 4104 & 4089 & 4846 & 3962 \\
\hline
\end{tabular}

Fuente: Servicio de Migraciones de la Federación Rusa, Федеральная Миграционная Служба 2009

Para cruzar las fronteras sin pasar por los puntos oficiales, los inmigrantes utilizan varias estrategias. Además, la frontera es bastante amplia (más de $1000 \mathrm{~km}$ en la provincia de Omsk y $1870 \mathrm{~km}$ en Orenburg oblast), pero los puntos fronterizos y los puestos aduaneros son escasos ${ }^{11}$. A finales de 1990, se produjo un cambio en la actitud de los gobiernos regionales de Rusia hacia la frontera con Kazajstán, debido a problemas como el aumento de tráfico de drogas y la criminalización de la zona. Esta evolución se explica por los cambios en la composición étnica de las regiones fronterizas debido a la afluencia de inmigrantes, el empeoramiento de la tensión interétnica, el comercio ilegal a gran escala y la propagación del Islam radical. La inestabilidad de los Estados de Asia Central y la posibilidad real de una insurgencia de los fundamentalistas islámicos del sur, plantean una amenaza particular. Como consecuencia, los gobiernos locales de las regiones fronterizas de Rusia exigen que las nuevas fronteras refuercen la protección y la seguridad (Codagnone, 1998).

La amplia frontera del sur se extiende desde el cruce de la frontera entre Rusia y China-Mongolia, hasta Rusia- China y Corea del Norte (RPDC) y comprende el Territorio de Altai, la región de Chita, la Región Autónoma Judía, el territorio Khabarovsk y el territorio Primorie. La situación fronteriza de esta amplia frontera puede ser reconocida como estable, por el momento, en el sentido de que, ni Japón ni China tienen la intención de recobrar por la fuerza los territorios que, consideran suyos. La violación sistemática de las leyes es frecuente en las fronteras de Rusia con China, Mongolia y Japón. Esta violación, a menudo, se debe a la actividad económica de la población de China y Mongolia y no tiene ninguna motivación política. En la frontera con Mongolia, los incidentes se producen debido a la demarcación inadecuada, a la escasez del control, o al desconocimiento del concepto de frontera por parte de la población local ${ }^{12}$ (Kunadze, 1999).

11 En provincia de Omsk, sólo hay siete puntos fronterizos. En provincia de Oremburgo, el Departamento de Fronteras ha identificado 600 rutas incontrolables para entrar en el territorio ruso. En segundo lugar, en la provincia de Omsk, por ejemplo, los puestos están ubicados lejos de la frontera (de 18 a 40 km o más). Por otra parte, el tráfico ferroviario entre Kazajstán y Rusia es un problema de difícil control.

12 Este es el caso de Mongolia en la frontera con las repúblicas rusas de Tuva y Buriatia Los Buriats y Tuvinians tienes estilos de vida muy próximos a los mongoles. El Gobierno de Mongolia reclamó, oficialmente los dos territorios Sin embargo, Rusia está dispuesta a mantener su régimen de fronteras sin realizar cesiones. 
Rusia y China comparten 2.500 kilómetros de frontera y 18 puestos de control, seis de ellos ubicados en la región de Primoroski. Por lo tanto, es natural ver a China como uno de los socios migratorios de Rusia. En los primeros años tras el desplome soviético, los ciudadanos chinos comenzaron la inmigración en masa a Rusia estableciendo, a lo largo del tiempo, un activo comercio de bienes de consumo a lo largo de la frontera ruso-china, especialmente en Siberia y en el Lejano Oriente. Hubo una genuina explosión migratoria. El número de ciudadanos chinos que entró en Rusia osciló en los últimos años entre 450.000 y 500.000 personas (Servicio de Migraciones de Rusia, 2009). Según la misma fuente, en las zonas fronterizas rusas con China, sólo 1.177 personas tenían, en 2009, residencia permanente. Sin lugar a dudas, el mayor flujo de inmigrantes chinos en Rusia, es irregular. Generalmente, las personas llegan a Rusia a través de Kazajstán, y después se dirigen hacia la parte central y occidental del país.

El aumento de los inmigrantes chinos en el Lejano Oriente de Rusia se produjo en paralelo a la afluencia de ciudadanos coreanos ${ }^{13}$, que se concentran principalmente en Primoroski krai, (33.000, del total de 125.000) donde existe una frontera compartida de 18.000 kilómetros, entre Rusia y Corea del Norte. Khabarovsk y Kamchatka son otras regiones del Lejano Oriente de Rusia, que tienen en su composición inmigrantes coreanos (Lee, 2002). El censo de 2002 no puede reflejar con claridad la situación real existente en las regiones fronterizas de Rusia y China. Según las estadísticas oficiales, el número de ciudadanos chinos en estas regiones es inferior a 10.000. (Golunov, $2007 \mathrm{~b}$ ).

\section{CAOS Y COMPLEJIDAD: MIGRACIÓN TRANSFRONTERIZA IRREGU- LAR EN RUSIA}

La frontera rusa es un instrumento clave utilizado por los inmigrantes irregulares que llegan a Rusia, principalmente de países de Asia y África. Con la relajación de las restricciones fronterizas se ha producido un aumento considerable, difícilmente cuantificable (Figura 3).

13 El grupo étnico coreano-chino, que se clasifica como una de las "55 nacionalidades minoritarias" por el gobierno chino, tiene casi dos millones de personas en China. 95\% de ellos, tienen su residencia en las tres provincias del noreste de China, antes llamada Manchuria, que comparte una larga frontera con el Extremo Oriente ruso. 
Figura 3. Intercepciones en las fronteras Rusas: 2000-2008

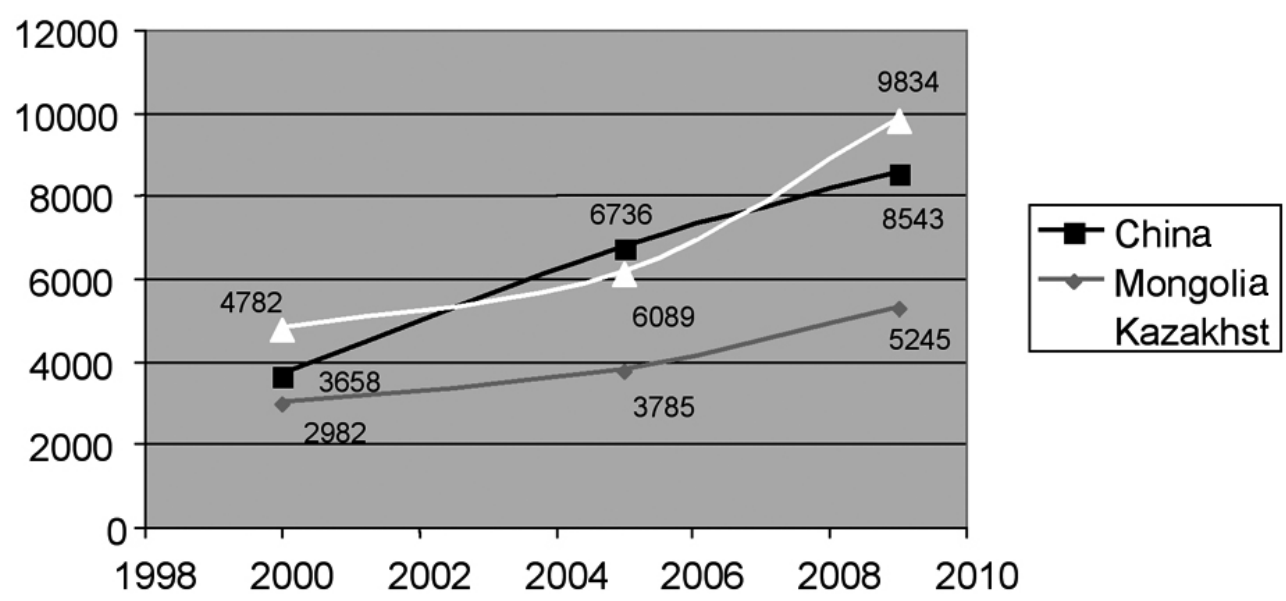

Fuente: Elaboración propia según el Informe de la Policía de Fronteras de Rusia, 2009

Pueden ser identificados tres grandes flujos de inmigrantes, que se diferencian según sus objetivos al llegar a Rusia: en primer lugar, los inmigrantes que buscan oportunidades de trabajo para mejorar sus ingresos y su calidad de vida. Se trata, sobre todo, de los inmigrantes que proceden de China y Vietnam y que entran en Rusia con documentos falsificados o cruzan las fronteras ilegalmente con identidades falsas.

En segundo lugar, se trata de los refugiados y solicitantes de asilo procedentes de regiones devastadas por la guerra, en situaciones de hambre y conflictos, siendo procedentes, principalmente, de Afganistán y de países africanos como Somalia, Etiopía, Angola. En esta categoría se incluyen los universitarios y graduados que llegaron a estudiar y no están dispuestos a regresar a sus países una vez acabados los estudios. Todas estas personas desean entrar en los países occidentales para encontrar empleo o para recibir el estatus oficial de refugiado.

Después de que Rusia firmara en 1993, la Convención (de 1951) de las Naciones Unidas para los refugiados, esta vía fue utilizada activamente por los inmigrantes de países asiáticos y africanos, con la intención de seguir avanzando hacia los países de la UE.

Por último, se trata de los migrantes en tránsito, que utilizan a Rusia como una vía para llegar a los países occidentales. Según el Ministerio del Interior ruso existe al menos medio millón de migrantes en tránsito desde Afganistán, China, Angola, Pakistán, India, Sri Lanka, Turquía, Etiopía, y otros países más próximos a Rusia14.

Así, pues, Rusia es principalmente un país de destino para los migrantes irregulares. En segundo lugar, es un país de tránsito para aquellos ciudadanos que están de

14 Nelegalnaya tranzitnaya migratsiya v Rossii [Migración irregular de tránsito en Rusia] (2002). (Moscow: Center for Political Information). [en Ruso]. 
camino hacia la Europa occidental y central, y en tercer lugar, un país de salida para los ciudadanos rusos que desean vivir o trabajar en otros países.

\subsection{INMIGRACIÓN IRREGULAR Y TRÁFICO HUMANO}

En el caso aquí estudiado, distinguimos dos tipos de rutas de inmigración irregular y de tráfico humano: un tipo de ruta de entrada y otro de ruta de salida. (Figura 4).

Figura 4. Rutas de migración irregular hacia y desde Rusia.

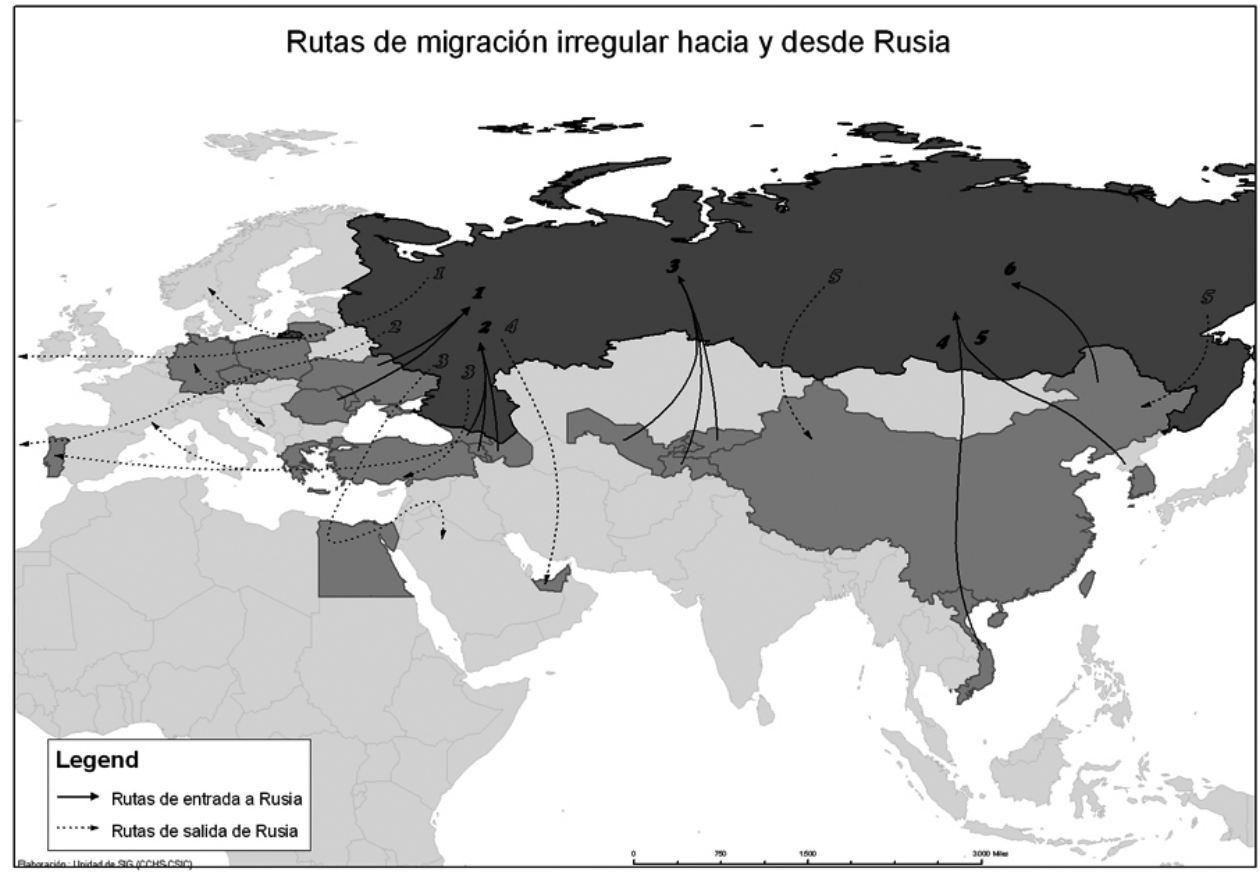

Fuente: Elaboración Laboratorio SIG CCHS, CSIC

Para cada tipo se identificaron varias rutas básicas utilizadas por los inmigrantes irregulares, las redes y los "transportistas" que captan las personas de dentro y de fuera de la Federación Rusa, para su explotación.

\subsubsection{LAS RUTAS DE ENTRADA EN EL TERRITORIO DE RUSIA}

Para la entrada en Rusia, se distinguen seis rutas principales:

1) La ruta europea: Rumania-Moldavia-Ucrania-Rusia

2) La ruta del Cáucaso: Armenia-Azerbaiyán y Georgia-Rusia

3) La ruta de Asia Central: Tayikistán - Uzbekistán - Kirguistán

4) La ruta de Vietnam del Sur, China y Mongolia

5) La ruta Sur-Oriente: Corea del Norte - China y Mongolia-Rusia

6) La ruta Este de China- Rusia 
La vía principal de los migrantes irregulares que llegan a Rusia, se realiza a través de las fronteras del sur, (Estados de Asia Central y del Cáucaso, con los que Rusia tiene acuerdos fronterizos).

Las redes de inmigración irregular se encargan de escoger las situaciones más favorables para la entrada en Rusia. De esta manera, buscan potenciales clientes en países y regiones con estructuras políticas y sociales fragmentadas, economías deterioradas y legalidad débil, donde las redes delictivas implicadas en el tráfico operan libremente, porque les está permitido.

Las rutas utilizadas para el contrabando y el tráfico de personas hacia Rusia se solapan, en parte, con las que se utilizan para el contrabando de drogas, cigarrillos y vehículos robados 15 .

Rusia es una de las principales puertas de entrada a Europa para los inmigrantes procedentes de Irak, Irán, Afganistán y muchos otros países asiáticos, incluida China. El mayor número de intentos de evitar los controles fronterizos se registra en el sector del Cáucaso, en las fronteras situadas entre Rusia, Azerbaiyán y Georgia (en 2009, tras el cierre fronterizo, se interceptaron 2.150 personas) - y el sector Nor-occidental - la frontera con Noruega, Finlandia, Estonia y Letonia (donde en 2009, fueron interceptadas 1.400 personas) ${ }^{16}$.

En la República de Kazajstán y en los sectores orientales (China, Mongolia y Corea del Norte), el número de intentos frustrados de cruzar la frontera llegó a $285.000^{17}$ personas, en 2009 .

Alrededor del 34\% de los inmigrantes irregulares que llegan a Rusia se encuentran detenidos en la frontera con Kazakhstan. La mayoría proceden de Afganistán, Bangladesh, Sri Lanka y Pakistán. De acuerdo con los guardias de fronteras, los inmigrantes que desean entrar en Rusia de modo irregular, poseen visados de Kirguistán, y, a veces, de Kazajstán. Los ciudadanos afganos, a menudo, tienen pasaportes falsificados, obtenidos en Tayikistán ${ }^{18}$.

Como señalamos, en la mayoría de los casos, estas personas que cruzan ilegalmente la frontera, están interesadas en Rusia, únicamente, como país de tránsito en su camino hacia la UE.

Por otra parte, el negocio criminal, para captar y transportar a los ciudadanos irregulares de países del sudeste de Asia, a Rusia o a Europa, es muy rentable. La fabricación de un pasaporte falso de Tayikistán para un ciudadano afgano, cuesta alrededor de 1.500 dólares (IOM, op.cit). Y mientras este negocio produce importantes ingresos, no hay un endurecimiento real de las leyes. La mayoría de estos incidentes implican violaciones de procedimiento. Incluso si consideramos este problema como un grave tema de seguridad fronteriza, su solución consistiría en la mejo-

15 Interpol http://www.interpol.int/Public/THB/PeopleSmuggling/Default.asp

16 International Centre for Migration Policy Development (ICMPD), 2010, p. 41.

17 Permanent Mission of the Russian Federation to the International Organization in Vienna

18 Border Guard Service of Russia 2009. 
ra de la calidad del control en los puntos fronterizos, y no en la fortificación de las fronteras estatales y el endurecimiento del régimen de entrada.

\subsubsection{LAS RUTAS DE SALIDA DEL TERRITORIO DE RUSIA:}

Para salir de Rusia se suelen utilizar las siguientes rutas, vinculadas sobre todo, al tráfico de personas, especialmente al tráfico y a la trata de mujeres.

1) La ruta del Báltico, que, a su vez, tiene dos ramificaciones:

a) Rusia-Lituania-Polonia-Alemania-Reino Unido (extendida hacia los EE.UU.),

b) Rusia-Lituania, extendida hacia los países nórdicos.

2) La ruta europea: Rusia-Polonia-República Checa-Alemania-Francia-España (ampliación a los EE.UU.)

3) La ruta de Europa del Este y del Sur de Rusia, Moldavia y Ucrania-GeorgiaTurquía y entre Egipto e Israel.

4) La ruta de Asia: Rusia y Emiratos Árabes

5) La Ruta China: Rusia (Siberia)-China, Rusia (Primorie) China Como resultado del deterioro de la economía y la apertura hacia el exterior, se ha producido un aumento en el tráfico irregular y de mujeres rusas hacia el Occidente. Existe una variedad de razones para explicar la presencia del tráfico y de la trata de personas en Rusia: la transparencia de las fronteras entre Rusia y los países de la CEI, el aumento de los flujos migratorios de dentro y de fuera de Rusia, y lo más destacado, el desarrollo interno del sistema de tráfico humano en Rusia, como uno de los más provechosos negocios de la esfera criminal (incluyendo el uso del trabajo forzoso en la globalización del crimen organizado debido a la existencia de canales estables para realizar la "entrega" de personas) (Hughes, 2002).

Las rutas del Báltico a través de Lituania, así como la que procede de Europa Central a través de Varsovia y Praga, constituyen los métodos más sencillos para el transporte de inmigrantes irregulares hacia Alemania, Escandinavia y Estados Unidos. La UE y Estados Unidos siguen siendo los principales países de destino para la trata de personas.

La ruta de tránsito que pasa por Georgia se ve facilitada por las fronteras débiles que el país tiene con Turquía.

Los expertos en el ámbito, (Golunov, op.cit b) señalan que la mayoría de los migrantes irregulares, entre ellos mujeres y niños se transportan a través de Georgia, a Turquía, Grecia y otros países mediterráneos con fines de explotación sexual y laboral.

Al mismo tiempo, las zonas de conflictos armados (Chechenia) o las regiones donde se encuentran las fuerzas militares y de mantenimiento de la paz, son consideradas verdaderos nidos y focos de tráfico para la explotación sexual en la región.

Las rutas a través de Egipto e Israel, o las que se dirigen hacia Oriente Medio, son también comunes para el tráfico de mujeres, que se captan de las regiones de sur de Rusia y se dirigen hacia los Emiratos Árabes Unidos con fines de explotación sexual ${ }^{19}$.

19 Human trafficking in the Russian Federation. Report UNICEF (2006), p. 25.

http://www.unicef.org/russia/ru_human_trafficking_eng.pdf 
El mayor flujo de tráfico para la explotación laboral se ha encontrado en los países con grandes diásporas rusas, como Alemania o Turquía donde los trabajadores son transportados principalmente para trabajar en condiciones de explotación en el sector de la construcción o en el sector agrícola.

El tráfico entre China y Rusia se divide entre las rutas situadas en las regiones fronterizas de China-Siberia y China- Región de Primoroski.

Los grupos delictivos chinos se han convertido en un gran problema para las autoridades policiales de Primoroski y Jabarovsk y de las regiones vecinas, donde el tráfico de ciudadanos chinos tiene un enorme potencial. Se trata de un negocio muy estable, orientado hacia la actividad a largo plazo, organizado más como una actividad empresarial de un grupo delictivo, que como una actividad tradicional. Por el contrario, el negocio de la trata de personas desde Rusia, no está marcado por la estabilidad, siendo más bien orientado hacia el beneficio inmediato.

Debido a la naturaleza clandestina e ilegal de la trata de personas, es difícil conocer el número exacto de víctimas pero las estimaciones (Tishkov et al. (2005, op.cit) apuntan a que más de 50.000 mujeres rusas son, anualmente, explotadas para la esclavitud sexual en Europa Occidental, Oriente Medio, Estados Unidos y Asia. Además, Ivakhniouk (op.cit) señala que los hombres, mujeres y niños de los países más pobres de la CEI son objeto de trata en Rusia, para su explotación laboral.

De acuerdo con la Misión Permanente de la Federación Rusa en la Organización Internacional de Viena, entre 2007 y 2008, se produjo la siguiente situación (Figura 5).

Figura 5. El tráfico de personas detectado en las fronteras de Rusia (número de personas)

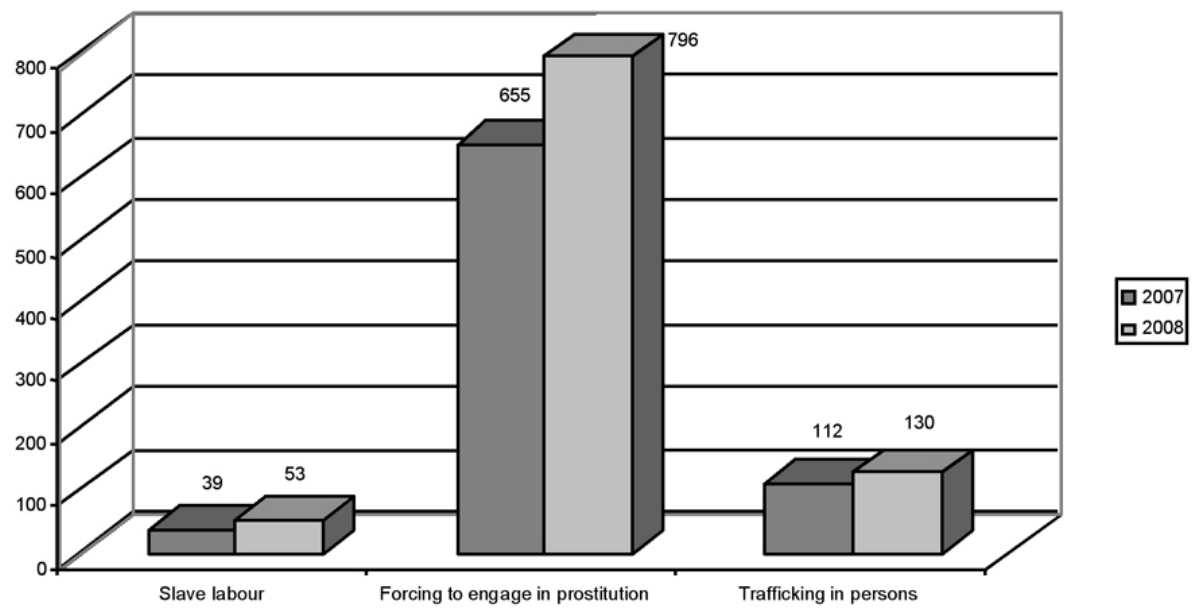

Fuente: Elaboración propia con datos de la Misión Permanente de la Federación Rusa en la Organización Internacional de Viena. http://www.rusmission.org/

\subsection{EL TRÁFICO DE DROGAS}

Un problema más que alarmante para la frontera rusa es su proceso de convergencia entre la migración irregular y el tráfico de drogas, que se desarrolló, sobre 
todo, desde la desmembración de la URSS. El contrabando a través de Rusia y Kazajstán, Rusia y Georgia, por una parte, y entre Rusia y Azerbaiyán por la otra, deriva de la producción de heroína en Afganistán, además de la producción de marihuana y hachís procedente de los países post-soviéticos de Asia Central ${ }^{20}$.

Y aunque el tráfico de marihuana (procedente de Ucrania y del Cáucaso) y de drogas sintéticas (que llegan de los países de la UE a través de Bielorrusia, Ucrania y los países Bálticos) tuvieron un impacto considerable en el mercado de drogas de Rusia, la heroína y el opio crudo (para su elaboración en heroína) procedente de Afganistán, siguen siendo los más peligrosos. En la década de 1990, este país se convirtió en el líder absoluto, como proveedor de los opiáceos. Actualmente, produce el $75-80 \%$ del volumen global (Trenin, Malasenko y Lievin, 2004).

El principal canal de tráfico de drogas en Rusia, lo constituye la frontera entre Rusia y Kazajstán, a través de la cual entra en Rusia, la marihuana y la heroína. En la actualidad, las estructuras legislativas interceptan sólo una parte insignificante de las drogas que llegan a Rusia. Con todo, cada año, se interceptan en las fronteras rusas, entre 1.000 y 1.500 kilos de heroína (Golunov, 2007op.cit a).

A pesar de que se señala que los grupos étnicos hayan desempeñado un papel importante en el contrabando de heroína de Rusia, la diferencia entre el enorme volumen de la migración procedente de Asia Central (1,5-2 millones personas al año) y el número de intercepciones de traficantes de heroína a través de la frontera entre Rusia y Kazajstán (unos 200 al año) no permite una asociación directa y clara del tráfico de drogas con los grupos de migrantes étnicos.

\section{CONCLUSIONES}

El artículo realizó una incursión por el laberinto migratorio de las fronteras rusas. Se analizaron las fronteras creadas después de la caída del régimen soviético, con sus principales características, relacionadas con las tensiones étnicas, la migración irregular y el tráfico de personas. Utilizando el ejemplo de Rusia, el artículo evidenció el vacío de información sobre los estudios fronterizos vinculados a la migración internacional.

La falta de conocimiento del fenómeno de la frontera y de las migraciones en Rusia, hizo que predominaran los enfoques alarmistas, mientras que los estereotipos creados contribuyeron a que las políticas de migración de Rusia fueran prácticamente desconocidas y contradictorias. La inexistencia de una estrategia migratoria y unos propósitos claramente definidos en la política de migración rusa, pueden explicarse por la falta de desarrollo que domina el panorama geopolítico, económico, demográfico o social.

Por otra parte, la crisis económica de los últimos 2-3 años destruyó la apariencia de prosperidad en Rusia: los precios del petróleo se desplomaron, los sectores bancarios entraron en crisis y el sector de la construcción, la principal fuente del empleo de los inmigrantes, se hundió, contribuyendo al recorte de los cupos de inmigrantes.

20 United Nations Office on Drugs and Crime http://www.unodc.org/ 
Aumentó la xenofobia y las empresas comenzaron a despedir a los inmigrantes, o, en algunos casos, simplemente dejaron de pagarles los sueldos ${ }^{21}$.

Se necesita profundizar un diálogo global con Rusia, tanto en los temas relacionados con la migración, como en el marco teórico de análisis de fronteras, la lucha contra la inmigración irregular y la trata de seres humanos, la migración de mano de obra y los aspectos sociales relevantes del cruce fronterizo y de la inmigración.

A los efectos de una mayor eficacia de las medidas encaminadas a la prevención de la migración irregular, se recomiendan los siguientes pasos de actuación:

1. En primer lugar, se debería conceder el libre derecho de cruzar la frontera a los habitantes que viven en las zonas fronterizas o en sus proximidades, y proporcionarles permisos especiales. Estos inmigrantes son irregulares sólo en el sentido técnico, debido a la imperfección de las leyes. En realidad, su vida es fronteriza, porque viven en las regiones limítrofes a las fronteras.

2. Intensificar las negociaciones de Rusia con los países fronterizos en la coordinación del régimen de fronteras estatales y los procedimientos de control.

3. Adoptar medidas encaminadas a mejorar la organización de los controles en los puntos fronterizos. Eso supondrá la reducción de los desvíos por parte de los ciudadanos extranjeros que respetan la ley.

Al mismo tiempo, se debe hacer hincapié en factores como la importancia del tráfico de drogas a través de las fronteras de Rusia, la subsanación eficaz de la escasez de mano de obra, el aumento de los ingresos y la reducción de las tensiones sociales. Hay que tener en cuenta, asimismo, que la migración por si sola no puede compensar la dramática pérdida de la población de Rusia. De acuerdo con la OIM, entre 2000 y 2050, Rusia exigiría una migración neta de 24,9 millones de personas para mantener el volumen de su población y una migración neta de 35,8 millones para mantener su población en edad laboral. (IOM, op.cit)

En todos estos aspectos migratorios del siglo XXI, la frontera juega un papel relevante. Por lo tanto, en el ámbito de los estudios científicos, sería importante detenerse en las lecciones que pueden extraerse de la geopolítica fronteriza en el estudio de las migraciones, en este caso de Rusia, y de los países de la antigua Unión Soviética.

\section{REFERENCIAS BIBLIOGRÁFICAS:}

BAGRANDZHIA, B.A. (2000): Vlianie subiektov $R F$ na reshenie voprosov federal'nogo znachenia. (La influencia del Estado de la Federación Rusa, para resolver los problemas de importancia federal). Moscú, El centro de Estudios Estratégicos.

BALATSKY, A. V. (1995): Vestnik Rossiiskoi Akademii Nauk. (El trabajo de los Inmigrantes económicos) Academia de Ciencias de Rusia, 872-880.

BATER, J. ARNOLD, L. (1996): Russia in the post-soviet scene, a geographical perspective. Political Geography, 17, 6, 751-752.

21 Moscow Times: 29 de Septiembre de 2009 
CODAGNONE, C. (1998): The New Migration in Rusia in the 1990. En: Koser, K. y Lutz, H. (ed.) The New Migration in Europe, London, Macmillan Press LTD.

ESKELINEN, H., LIIKANEN, I., AND OKSA, J. (1999): Introduction En: Åskelinen, H., Liikanen, I. \& Oksa, J. (eds.) Curtains of Iron and Gold. Reconstructing Borders and Scales of Interaction. Aldershot, Ashgate.

FLYNN, M. (2004): Migrant Resettlement in the Russian Federation: Reconstructing Homes and Homelands. London, Anthem Press.

FRANKLIN, S. Y WIDDIS, E. (eds.) (2004) National Identity in Russian Culture: An Introduction, Cambridge, University Press.

GIDDENS, A. (1987): The Nation-State and Violence. Berkeley, University of California Press.

GIELIS, R. (2009): Borders make the difference: migrant transnationalism as a border experience. Tijdschrift voor economische en sociale geografie, Vol. 10, (5) 598-609.

GOLUNOV, S. (2007): (a). Drug-trafficking through Russia's Post-Soviet Borders: Problems, Misperceptions and Countermeasures. Acta Slavica Lapónica, 24, 24-46.

GOLUNOV, S. (2007): (b). Ethnic Migration: A Challenge to Russian Border Security? PONARS Memo, 432 Center for Strategic and International Studies.

HELENIAK, T. (2003): The 2002 Census in Russia: Preliminary Results. Eurasian Geography and Economics, 44, (6) 430- 442.

HELENIAK, T. (2002): Migration Dilemmas Haunt Post-Soviet Russia. Migration Information Source, Migration Policy Institute, (5).

HIRSCH, F. (2000): Toward an Empire of Nations: Border-Making and the Formation of Soviet National Identities. Russian Review, 69, (2), 201-226.

HOUTUM, H. (2005): The Geopolitics of Borders and Boundaries. Geopolitics, 10, (4) 672-679.

HOUTUM, H.; NAERSSEN T. (2002): Bordering, Ordering and Othering. In Tijdschrift voor Economische en Sociale Geografie, 93, (2)125-136.

HUGHES, D. (2002): Trafficking for Sexual Exploitation: The Case of the Russian Federation. IOM Migration Research Series, 7 June 2002, 9.

KONONENKO, V. (2004): What's new about today's EU-Russia border? Working Paper, 50 Ulkopoliittinen Instituutti (UPI), The Finnish Institute of International Affairs (FIIA).

http://www.upi-fiia.fi

KUNADZE, G. (1999): Border problems between Russia and its Neighbours. Stable for now, but Stubborn in the long run. En Arbatov, A.G., Hartelius, D., Schmemann, A. Eurasia in the 21st century: the total security environment (Volume 3) Ed., Rozman, G., Nosov, M. East West Institute.

KUZIO, T. (1997): Borders, symbolism and nation-state building: Ukraine and Russia. Geopolitics, 2, 36 - 56.

IOM, (2009): Migration in the Russian Federation: A Country Profile (2008). Switzerland.

http://publications.iom.int/bookstore/index.php?main_page=product_info\&cPath=4 1_42\&products_id=494

IVAKHNYOUK, I. (2006): Migration in the CIS Region: Common Problems and Mutual Benefits. An expert paper presented at the International Symposium on 
International Migration and Development, United Nations Population Division, 2830 June 2006, Turin, Italia.

LEE, J. (2002): Migration, Ethnicity and Citizenship: Ethnic-Korean Returnees in the Russian Far East. Asia Culture Forum, Inha University.

LEVITT, P., DE WIND, J., VERTOVEC S. (2003): International Perspectives on Transnational Migration: An Introduction. International Migration Review, 37, (3) 565-575.

O'LOUGHLIN, J., VAN DER WUSTEN, H. (1993): The new political geography of Eastern Europe, London, Belhaven Press.

MARCU, S. (2007): La geopolítica de la Rusia postsoviética: desintegración, renacimiento de una potencia y nuevas corrientes de pensamiento geopolítico. Scripta Nova, vol. XI, 253, (11), 229-255. http://www.ub.es/geocrit/sn/sn-253.htm

PAASI, A. (2003): Boundaries in a Globalizing Word. En Anderson, K. Handbook of Cultural Geography, 462, London, Sage.

PAASI, A. (1999): Boundaries as Social Practice and Discourse: The Finnish - Russian Border. Regional Studies 33(7), 669-680.

PILKINGTON, H. (1998): Migration, Displacement Identity in Post-Soviet Russia. London, Routledge.

PRESCOTT, J.V. (1987): Political frontiers and boundaries. London, Allen and Unwin.

RUMFORD, C. (2006): Theorizing Borders. European Journal of Social Theory, 9, (2), 155-170.

TISHKOV, V. A. (Ed.) (1996): Migratsii $i$ Novye Diaspory v Postsovetskikh Gosudarstvakh. (Migraciones y Nuevas Diásporas en los Estados post soviéticos). Academia de Ciencias de Rusia, Instituto de Etnología y Antropología, Moscú.

TISHKOV, V., ZAYINCHKOVSKAYA, Z., VITKOVSKAYA, G. (2005): Migration in the Countries of the Former Soviet Union. Policy Analysis and Research Programme of the Global Commission on International Migration.

TRENIN, D. (1998): Baltiysky shans (The Baltic Chance. Baltic Countries, Russia and the West in Emerging Big Europe). Moscow Carnegie Center.

TRENIN, D., MALASENKO, A. Y LIEVIN, A. (2004): Russia's restless Frontier: the Chechnya factor in Post Soviet Russia. Washington D.C. Carnegie Endowment for International Peace.

TSUDA, T. (2003): Strangers in the Ethnic Homeland. New York, Columbia University Press.

VARDOMSKI, L. (2002): Russia's new borders in the context of regional cooperation in the post Soviet space. Russian Regional Perspectives Journal 1, (2) The International Institute for Strategic Studies http://www.iiss.org/programmes/russiaand-eurasia/copyof-russian-regional-perspectives-journal/copyof-rrp-volume-1issue-2/russias-foreign-and-security-policy-in-central-asia/

VITKOVSKAYA, G. (1996): Relocation to Russia from the states of Central Asia: understanding the decision to migrate. En Azrael, J. R., Payin, E. A. (Eds.), Cooperation and Conflict in the Former Soviet Union: Implications for Migration, Santa Monica, California, RAND.

VORONINA, N. (2006): Outlook on Migration Policy Reform in Russia: Contemporary Challenges and Political Paradoxes. En Rios, Roger Rodríguez, Migration 
Perspectives. Eastern Europe and Central Asia. Planning and Managing Labour Migration, Vienna, IOM, 71-90.

ZACHAROVA, O., MINDAGULOV V., RYBAKOVSKY, L. (1994): Illegal Migration in Border Territories of the Far East. Sotsiologicheskie issledovaniia 12, 11-21.

ZAIONCHKOVSKAYA, Z. (1994): Migratsiya Naseleniya i Rynok Truda v Rossii (Population Migration and the Labor Market in Russia). Institut Narodnokhozyaistvennogo Prognozirovaniya, Academia de Rusia, Instituto de Estudios Económicos. 